\title{
Diagnosis of Heart Valve Disorders through Trapezoidal Features and Hybrid Classifier
}

\author{
Fatemeh Safara, Shyamala Doraisamy, Azreen Azman, Azrul Jantan, and Sri Ranga
}

\begin{abstract}
Numerous studies are being conducted in recent years focusing on phonocardiographic (PCG) signals due to their capability to characterize heart sounds. These characteristics can be exploited in developing computer-aided auscultation system as a complementary tool for clinicians in diagnosis of cardiovascular disorders. This study proposes a new type of features to distinguish five categories of heart sounds, including normal, mitral stenosis, mitral regurgitation, aortic stenosis, and aortic regurgitation. PCG signals were collected from online resources and training CDs. Wavelet packet transform was utilized for heart sound analysis as opposed to discrete wavelet transform that has been extensively used in the previous studies. Then, trapezoidal function was calculated for deriving feature vectors. A hybrid classifier was designed composing of three types of classifiers, multilayer perceptron (MLP) artificial neural network, k-nearest neighbor $(\mathrm{KNN})$, and support vector machine (SVM), to classify feature vectors. The promising results demonstrate the effectiveness of the proposed trapezoidal features and hybrid classifier for heart sound classification.
\end{abstract}

Index Terms-Feature extraction, heart murmur, hybrid classification, trapezoidal function, wavelet packet transform.

\section{INTRODUCTION}

Heart sound is a biomedical signal with valuable diagnostic information about structural abnormality of the heart valves and associated great vessels. However, making diagnosis based on the sounds heard by means of a stethoscope is a difficult skill. Signal processing techniques can be employed to process the phonocardiographic signals (PCG) towards improving the accuracy of diagnosis. Phonocardiography is the registration of sound vibrations of heart and blood flow that has a capability of presenting various heart valve disorders.

Focus of this study is on pathological murmurs, which require medical attention. Murmurs are high frequency and noise like sounds produced from the turbulence on the blood moving from a narrow cardiac valves or reflow from the atrioventricular valves. Mitral regurgitation, mitral stenosis, aortic stenosis, and aortic regurgitation are amongst the most common murmurs. Fig. 1 presents the PCG of a normal heart sound and Fig. 2 illustrates samples of the pathological murmurs of this study. The differences are clearly shown and can be exploited to differentiate the murmurs from each other

Manuscript received May 10, 2013; revised July 15, 2013.

Fatemeh Safara, Shyamala Doraisamy, Azreen Azman, and Azrul Jantan are with the faculty of Computer Science and Information Technology, University Putra Malaysia, 43400 Serdang, Malaysia (e-mail: fsafara@yahoo.com,_ shyamala@fsktm.upm.edu.my, azreen@fsktm.upm.edu.my, azrul@fsktm.upm.edu.my).

Sri Ranga is with the Cardiology department, Serdang Hospital, 43000 Kajang, Malaysia (e-mail: sriranga71@hotmail.com). and from the normal heart sounds.

PCG signal processing is one of the active research areas [1]-[3]. A considerable body of these researches is dedicated to heart sound classification. Two major steps are required preceding the actual classification: preprocessing and feature generation.

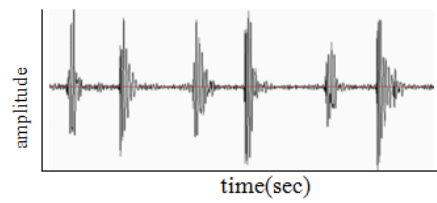

Fig. 1. Phonocardiogram of a normal heart sound.

The first major step in the whole classification process is preprocessing. Filtering, normalization, and segmentation are the techniques usually used in this step. Extraction of localized features depends on precise knowledge about the timing of the heart cycles. It can be achieved through segmentation, i.e. identification of the first and the second heart sound ( 1 1 \& S2). There are a number of researches that were addressed PCG segmentation [4], [5], although some works were reported for classifying heart sound without segmentation [6]-[8].

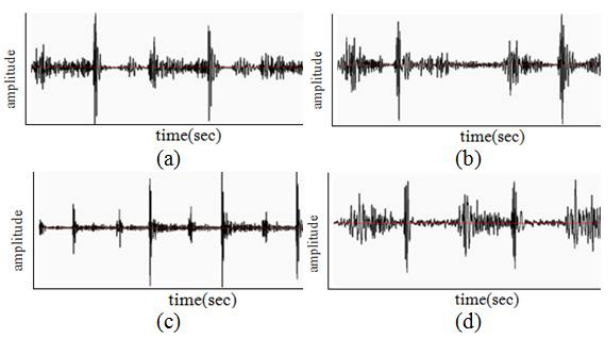

Fig. 2. Four phonocardiogram samples of murmurs of this study; (a) mitral regurgitation, (b) mitral stenosis, (c) aortic regurgitation, and (d) aortic stenosis.

In order to extract discriminant features, an appropriate signal analysis technique is required. Fourier and wavelet transform are broadly used signal analysis techniques in the recent studies. Fourier transform is a powerful technique to analyze the frequency components in PCG signals, but it suffers from the time-frequency resolution compromise. In the medical applications though wavelet approach was found to be the best alternative to the Fourier approach in analyzing the transient and non-linear signals such as heart sound, because it provides adaptive resolution in both frequency and time domain. In this paper, wavelet packet transform (WPT) was chosen from the wavelet family of transformations to analyze PCG signals.

Feature generation is the second major step preceding the actual classification that largely affects the result. A number of features proposed based on wavelet transform to 
differentiate murmurs from normal heart sounds. Ahlstrom et al. [9], applied techniques such as entropy, energy, recurrence quantification analysis and Fractal dimension to generate features, and classify systolic murmurs. Choi et al. [10] defined two features, meanWPE and stdWPE, based on wavelet packet energy (WPE); and the contribution of these two types of features was evaluated using accuracy achieved by support vector machine (SVM) classifier. In another investigation by Choi et al. [2], diagnostic features were proposed on WPT to achieve better accuracy for detecting regurgitation type of murmurs, namely the ratios of the wavelet energy and entropy, the maximum peak frequency, the position index of the wavelet packet coefficient corresponding to the maximum peak frequency. In this paper, trapezoidal function is proposed to generate features with the capability of differentiating five types of heart sounds and murmurs including normal heart sounds, AR, AS, MR, and MS.

The type of heart disorder would be determined by classifying heart sound based on the generated features. Multilayer perceptron (MLP) artificial neural network, K-nearest neighbor (KNN), and SVM, are promising classifiers that have been extensively used for heart sound classification. These classifiers were combined into a hybrid classifier to differentiate heart sounds of this study.

The reminder of this paper is organized as follows: In Section II, data preparation and full details of the background theory of WPT and feature generation are explained as well as hybrid classification. The result of classification through proposed hybrid classifier and proposed feature set is presented in Section III. Section IV provides a discussion about the results, and concludes the paper with comments for future studies.

\section{MATERIALS AND Methods}

\section{A. Data Preparation}

Data collection includes a set of 350 heart cycles comprising 50 normal and 300 pathological heart sounds (80 $\mathrm{MR}, 50 \mathrm{AR}, 70 \mathrm{MS}$ and $100 \mathrm{AS}$ ) extracted from online resources ${ }^{123}$ and auscultation training CDs. Resampling, filtering, normalization and segmentation were performed in preprocessing step to prepare PCG signals to be manipulated effectively in following steps.

Sampling frequencies of 4, 5, 10, 20, 25, 40 and $50 \mathrm{kHz}$ can be seen among the heart sounds collected. In order to remove the heterogeneity of the collected PCG signals, the original signals in the core frequency sampling were mapped into a new $4 \mathrm{kHz}$ frequency. The signal was considered as a time-series and new samples are produced by application of the truncated Sinc function interpolation. PCG signals were then band-pass filtered with $25-700 \mathrm{~Hz}$ cut-off frequencies which is the frequency range of heart sounds [10]. Finite response filter (FIR) with the minimum order Kaiser Window was used to remove unwanted noises. Later on, PCG signals were normalized using the following equation:

\footnotetext{
${ }^{1}$ Texas Heart Institute, Texas Heart Sound Database

${ }^{2}$ University of Michigan, Heart Sound and Murmur Library

${ }^{3}$ University of Dundee, Heart Sound \& Murmurs
}

$$
x_{\text {norm }}(n)=\frac{x(n)}{\max (|x(n)|)}
$$

where $n$ is the number of data points, $x(n)$ is the heart sound signal, and $x_{\text {norm }}(n)$ is the normalized signal to be used in this study.

After that, all PCG signals were segmented into their cycles and each of the cycles was further segmented into its systole and diastole manually. Therefore, two segments for each heart cycle were prepared to be used in the following step for generating features.

\section{B. Feature Generation}

Wavelet packet transform is a powerful tool in analyzing non-linear signals such as PCG. Discrete wavelet transform (DWT) decomposes a signal into approximation and detail coefficient. In successive steps, the approximation coefficients are decomposed only. However, WPT is an extension to DWT that at each step both the approximation and detail coefficients are decomposed into approximation and detail coefficients, as shown in Fig. 3, and more opportunities are provided for feature generation [14].

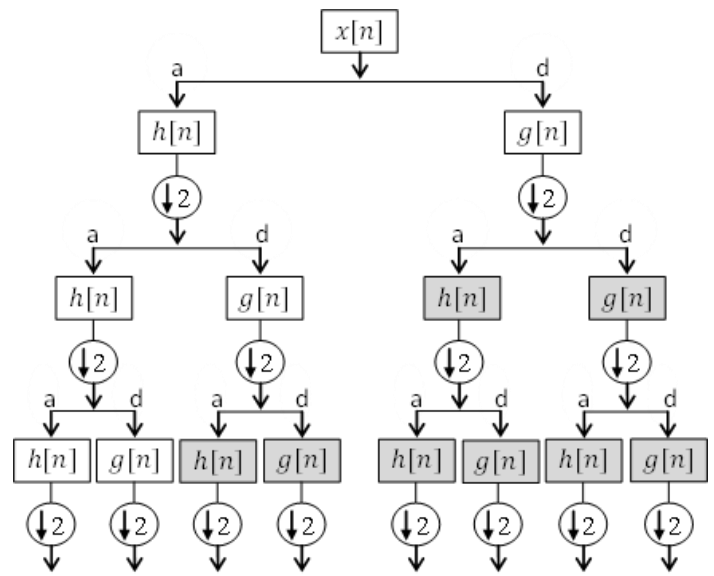

Fig. 3. Wavelet packet tree with corresponding high-pass and low pass filters (a=approximation coefficients, $d=$ detail coefficients). The shaded nodes indicate the node not to be produced by DWT

This structure can be further expanded to reach to a suitable frequency resolution for the application at hand. The WPT of a signal $x(t)$ is defined as follows:

$$
x_{p}^{n, j}=2^{j_{1 / 2}} \int_{R} x(t) \psi_{n}\left(2^{-j_{t}}-p\right) d t, 0 \leq n \leq 2^{s}-1
$$

where $n$ is the channel number, $S$ is the maximum level of decomposition, $j$ is the current level of decomposition, or socalled scale parameter, $p$ is the position parameter, $\psi_{n}(t)$ is wavelet packet function or mother wavelet. If signal $x(t)$ decomposes by WPT, $2^{S}$ sequences can be obtained in the Sth level. Given $h(i)$ and $g(i)$ are high-pass and low-pass filters, the decomposition equation for WPT is as follows:

$$
\begin{aligned}
& x_{k}^{2 n, j+1}=\sum_{p \in z} h(p-2 k) x_{p}^{n, j} \\
& x_{k}^{2 n+1, j+1}=\sum_{p \in z} g(p-2 k) x_{p}^{n, j}
\end{aligned}
$$

In this paper, appropriate representations of heart sounds and murmurs were acquired by WPT. To find a proper level of decomposition, level five to eight of wavelet packet tree 
were examined with different types of mother wavelets. The Symlets, Daubiches, Coiflets and Mayer were tried as potential mother wavelets. Level 6 of decomposition with Daubiches8 was found to be the best. After that, trapezoidal function was calculated from the coefficients of the first 22 terminal nodes that cover the frequency range of the heart sounds. Frequency range (FR) of each node of the wavelet packet tree can be obtained through the following equation:

$$
F R\left(x(t)=\frac{0.5 \times F S(x(t))}{2^{L}}\right.
$$

where $x(t)$ is the signal of study, 0.5 is the Nyquist rate, $F S$ is sampling frequency of the signal under consideration, $L$ is the decomposition level. Therefore, frequency range of heart sounds can be captures by only the first 22 nodes at level 6 of decomposition where the sampling frequency is $4 \mathrm{kHz}$. The trapezoidal function approximates the region under the graph of the one dimensional signal $x(t)$ in interval $[a, b]$ as follows:

$$
\int_{a}^{b} x(t) d x \approx(b-a) \frac{x(a)+x(b)}{2}
$$

Therefore, trapezoidal function can be used to approximate the area under the curve of PCG signals represented by WPT. In other word, trapezoidal function can be utilized to approximate the area under the WPT coefficients as feature for classification. The procedure explained above for feature generation was repeated for each systole and diastole segment of a heart cycle and therefore a feature vector comprising 44 features was obtained. Principal component analysis (PCA) was applied on each feature vector to reduce the number of features to 16 . The final feature vectors are fed into classifier.

\section{Constructing the Hybrid Classifier}

The main idea behind ensemble classification is the interest in exploiting advantage of various classifiers at their disposal to come up with a consensus result. Diversity in ensemble classification can be considered in: 1) selecting different classifiers also called hybrid classification; 2) training one classifier on different portions of the data; 3) using one classifier with different parameter specifications, and 4) using different feature sets. In this paper, approach 1 and 3 were explored. For this study, KNN, SVM and MLP with different specifications were examined and reasonable results were achieved. However, to further improve the classification performance these classifiers were combined into a hybrid classifier as shown in Fig.4; better accuracy was attained in compare with that of the individual classifiers. The specification of each classifier is as follows:

SVM: SVM with RBF kernel function was included in the hybrid classifier. In this type of SVM two parameters should be tuned including regularization parameter and degree of polynomial kernel. Here SVM-RBF with the regularization parameter of 150 and polynomial kernel of the degree 0.001 were used.

KNN: To find the best parameter $\mathrm{K}$ for the given train dataset, $\mathrm{K}$ is changed from 1 to 20 and the accuracy was calculated in each step. The optimum $\mathrm{K}$ is determined as 10 .

MLP: Three MLP were used whereby the maximum epoch number was chosen to be 500 for each MLP. Specification for each MLP is as follows. MLP-RBF1: one hidden layer with 20 neurons, tansig and logsig as the activation functions of the hidden layer and output layer, respectively. MLP-RBF2: one hidden layer with 26 neurons, tansig as the activation functions for both layers. MLP-RBF3: one hidden layer with 22 neurons, logsig as the activation functions for both layers.

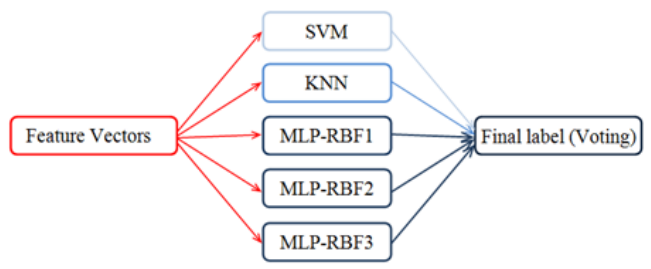

Fig. 4. Hybrid classifier composed of different classifiers (three MLP-RBFs with different specification, KNN, and SVM).

Final decision was made based on the median and mean values of all outputs. If the median and the mean of the voted values were not equal, the classifier with maximum repetition was chosen as the overall classification result. Otherwise, the maximum accuracy was chosen as the final decision.

\section{RESUlTS AND DISCUSSION}

An overview of the processes followed in this study is illustrated in Fig. 5.

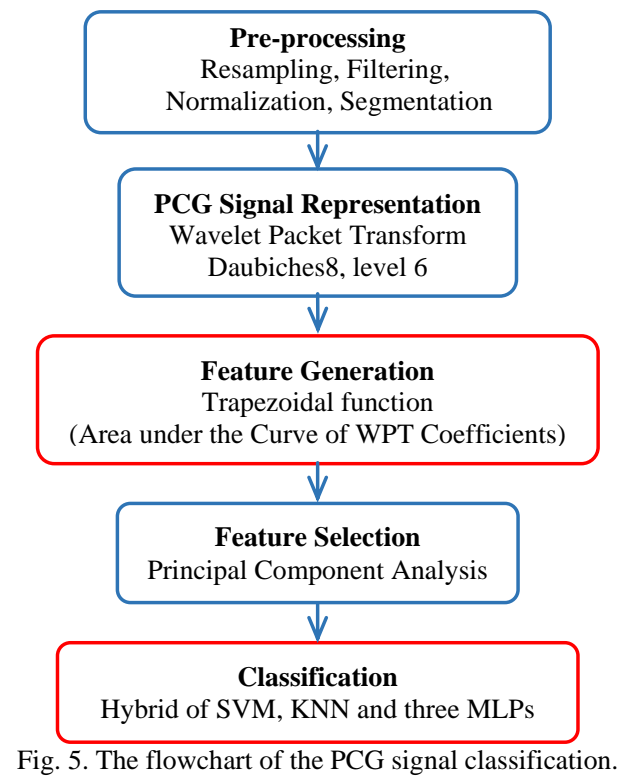

After preparing heart sounds in the preprocessing step, a feature vector including 44 features was constructed for each heart cycle in feature generation step. In the next step, the dimensionality of feature vector reduced to 16 in order to decrease the computation complexity of classification. Three types of classifiers were examined to classify the heart sounds and murmurs and the best specification of each of the classifiers was determined. Then a hybrid classifier including SVM, KNN and three MLP was designed to improve the overall accuracy of the classification. The results were better than or equal to the results of each constructive classifier. The best sensitivity of $96.74 \%$ and specificity of $97.85 \%$ were achieved for differentiating five types of heart sounds and murmurs including normal heart sound, AR, AS, MR and MS.

The results obtained in this study are comparable with the 
past reported researches. A vast amount of studies has been conducted to generate features based on wavelet transform family, particularly using continuous and discrete wavelet transform. Most of these researches were dedicated to distinguish normal heart sounds from murmurs and the rest were tried to distinguish different types of murmurs. Ahlstrom et al. [9] proposed different linear and non-linear features based on DWT, and classified a set of systolic murmurs with $86 \%$ accuracy. In another work by Ahlstrom et al. [15], recurrence quantification analysis was proposed to differentiate aortic stenosis from innocent murmurs, and $90 \%$ sensitivity and $88 \%$ specificity were achieved. Choi et al. [16] presented autoregressive power spectral density curves of DWT as a new feature for PCG signals classification and achieved $99.5 \%$ sensitivity and $99.9 \%$ specificity to classify normal heart sounds from pathological ones. Babaei et al. [17] utilized the main statistical characteristics of PCG signals such as mean and standard deviation in different level of DWT decomposition and acquired 94.24\% accuracy for classifying AR, AS and PS (pulmonary stenosis). A framework was proposed by Yuenyong et al. [6] based on DWT and without segmentation, for heart sound classification and gained $92 \%$ accuracy to classify a number of different heart valve disorders. Studies were reported by Choi et al. [2], [10] based on WPT for classifying heart sounds. In [10] two features were proposed to distinguish normal heart sounds from murmurs where $96 \%$ sensitivity and $100 \%$ specificity were attained. In a similar work [2], energy and entropy of WPT were utilized to differentiate normal heart sounds from regurgitation types of murmurs with $99.78 \%$ specificity and $99.43 \%$ sensitivity.

The murmurs that were considered in this study are the most common types of murmurs; however, in future works the heart murmur categories would be expanded to include other types of heart valve disorders. The promising results achieved in this study indicate the discriminatory power of the trapezoidal feature proposed and effectiveness of the hybrid classification. Heart sounds classified in this study were collected from internet resources and training CDs, which usually are clean from background noises. An important extension of this study is to examine the proposed features on real heart sounds.

In addition, real heart sounds should be taken from patients and the proposed feature and classifier will be examined to classify real heart sounds.

\section{REFERENCES}

[1] H. Naseri and M. R. Homaeinezhad, "Detection and boundary identification of phonocardiogram sounds using an expert frequency-energy based metric," Annals of biomedical engineering, vol. 41, no. 2, pp. 279-92, Feb. 2013.

[2] S. Choi, Y. Shin, and H.-K. Park, "Selection of wavelet packet measures for insufficiency murmur identification," Expert Systems with Applications, vol. 38, no. 4, pp. 4264-4271, Apr. 2011

[3] F. Safara, S. Doraisamy, A. Azman, A. Jantan, and S. Ranga, "Wavelet Packet Entropy for Heart Murmurs Classification," Advances in Bioinformatics, vol. 2012, pp. 1-6, 2012.

[4] M. R. Homaeinezhad, S. A. Atyabi, E. Daneshvar, A. Ghaffari, and M. Tahmasebi, "Discrete wavelet-aided delineation of PCG signal events via analysis of an area curve length-based decision statistic," Cardiovascular engineering, vol. 10, no. 4, pp. 218-34, Dec. 2010.

[5] M. R. Homaeinezhad, S. A. Atyabi, E. Deneshvar, A. Ghaffari, and M. Tahmasebi, "Optimal delineation of PCG sounds via false-alarm bounded segmentation of a wavelet-based principal components analyzed metric," International Journal for Numerical Methods in Biomedical Engineering, vol. 27, no. 11, pp. 1711-1739, 2011.
[6] S. Yuenyong, A. Nishihara, W. Kongprawechnon, K. Tungpimolrut, Y. Sumeth, N. Akinori, K. Waree, and T. Kanokvate, "A framework for automatic heart sound analysis without segmentation," Biomedical engineering online, vol. 10, no. 13, Jan. 2011.

[7] S. Jabbari, "A Time-Frequency Approach for Discrimination of Heart Murmurs," Journal of Signal and Information Processing, vol. 2, no. 3, pp. 232-237, 2011.

[8] Y. Chen, S. Wang, C.-H. Shen, and F. K. Choy, "Matrix decomposition based feature extraction for murmur classification," Medical engineering \& physics, vol. 34, no. 6, pp. 756-61, Jul. 2012.

[9] C. Ahlstrom, P. Hult, P. Rask, J.-E. Karlsson, E. Nylander, U. Dahlström, and P. Ask, "Feature extraction for systolic heart murmur classification," Annals of biomedical engineering, vol. 34, no. 11, pp. 1666-77, Nov. 2006

[10] S. Choi, "Detection of valvular heart disorders using wavelet packet decomposition and support vector machine," Expert Systems with Applications, vol. 35, no. 4, pp. 1679-1687, Nov. 2008.

[11] Texas Heart Sound Database. (2011). Texas Heart Institute. [Online] Available: http://www.tmc.ed/thi/anatomy2.html

[12] R. S. MacWalter, "Heart Sound \& Murmurs," University of Dundee, Ninewells Hospital \& Medical School, Dundee, Scotland, 2011.

[13] Heart Sound and Murmur Library, University of Michigan, 2011.

[14] P. I. J. Keeton and F. S. Schlindwein, "Application of wavelets in Doppler ultrasound Application of wavelets," Sensor review, vol. 17, no. 1 , pp. $38-45,1997$.

[15] C. Ahlstrom, K. Höglund, and P. Hult, "Distinguishing innocent murmurs from murmurs caused by aortic stenosis by recurrence quantification analysis," International journal of biological and medical sciences, vol. 1, no. 3, pp. 213-218, 2006.

[16] S. Choi and Z. Jiang, "Cardiac sound murmurs classification with autoregressive spectral analysis and multi-support vector machine technique," Computers in biology and medicine, vol. 40, no. 1, pp. 8-20, Jan. 2010.

[17] S. Babaei and A. Geranmayeh, "Heart sound reproduction based on neural network classification of cardiac valve disorders using wavelet transforms of PCG signals," Computers in biology and medicine, vol. 39, no. 1, pp. 8-15, Jan. 2009.

Fatemeh Safara is a Ph.D. candidate at the Faculty of Computer Science and Information Technology, University Putra Malaysia (UPM). She received the B.S. degree in applied mathematics and M.S. degree in computer engineering from Tarbiat Modares University, Tehran, Iran. Her research interests include audio and image processing, audio mining and biometrics.

Shyamala Doraisamy is an associate professor at the Faculty of Computer Science and Information Technology, University Putra Malaysia (UPM). She obtained her $\mathrm{PhD}$ in the area of music information Retrieval from Imperial College London in 2004. Her area of interest covers information retrieval, audio mining and multimedia audio technologies. She is currently the head of the Multimedia Information Retrieval Research Group at UPM.

Azreen azman completed his bachelor degree in 1999. He received Ph.D in computing science specializing in Information Retrieval in the University of Glasgow, Scotland in September 2007. He later joined University Putra Malaysia in May 2009 as a senior lecturer in the Department of Multimedia, Faculty of Computer Science and Information Technology. He is also a member of Digital Information Computation and Retrieval research group. His research interests are information retrieval and text mining.

Azrul Hazri Jantan received his $\mathrm{PhD}$ degree in Universiti Sains Malaysia, Penang in 2009. He is working as Senior Lecturer in Faculty of Computer Science and Information Technology, Universiti Putra Malaysia. His current research interests include web engineering and hypermedia design and development.

Sri Ranga was obtained his basic degree in Medicine from the National University of Malaysia in 1996 with first class honors and won the gold medal for medicine. He completed his master Masters in Internal Medcine from the National University of Malaysia in 2003. He was awarded the first place in his batch and won the prestigious Khalid Kadir award. In 2007, he joined cardiology training at Serdang Hospital as its first fellow. $\mathrm{He}$ completed training in 2009 and was attached to National Heart Institute (IJN) of Malaysia. His main area of interest is interventional cardiology especially primary PCI and establishment of a STEMI network within regional hospitals. 